\title{
Exploring the accessible frequency range of phase-resolved ferromagnetic resonance detected with $\mathbf{x}$-rays
}

\author{
P. Warnicke, ${ }^{1, a)}$ R. Knut, ${ }^{2}$ E. Wahlström, ${ }^{3}$ O. Karis, ${ }^{2}$ W. E. Bailey, ${ }^{4}$ and D. A. Arena ${ }^{1}$ \\ ${ }^{1}$ National Synchrotron Light Source, Brookhaven National Laboratory, Upton, New York 11973, USA \\ ${ }^{2}$ Department of Physics, Uppsala University, Uppsala, Sweden \\ ${ }^{3}$ Department of Physics, Norwegian University of Science and Technology, Trondheim, Norway \\ ${ }^{4}$ Materials Science and Engineering Program, Department of Applied Physics and Applied Mathematics, \\ Columbia University, New York,, New York 10027, USA
}

(Received 4 April 2012; accepted 29 November 2012; published online 16 January 2013)

\begin{abstract}
We present time- and element-resolved measurements of the magnetization dynamics in a ferromagnetic trilayer structure. A pump-probe scheme was utilized with a microwave magnetic excitation field phase-locked to the photon bunches and x-ray magnetic circular dichroism in transmission geometry. Using a relatively large photon bunch length with a full width at half maximum of $650 \mathrm{ps}$, the precessional motion of the magnetization was resolved up to frequencies of $2.5 \mathrm{GHz}$, thereby enabling sampling at frequencies significantly above the inverse bunch length. By simulating the experimental data with a numerical model based on a forced harmonic oscillator, we obtain good correlation between the two. The model, which includes timing jitter analysis, is used to predict the accessible frequency range of x-ray detected ferromagnetic resonance. (C) 2013 American Institute of Physics. [http://dx.doi.org/10.1063/1.4772613]
\end{abstract}

\section{INTRODUCTION}

A preferred technique for examining magnetization dynamics at $\mathrm{GHz}$ frequencies is ferromagnetic resonance (FMR), which has been used extensively to examine issues such as damping mechanisms in elemental and compound ferromagnets. ${ }^{1-4}$ FMR is particularly well suited for examining magnetization dynamics as the energy scale of the microwave excitation naturally matches with the precessional motion of the moments in many ferromagnetic materials. Recently, the technique has been expanded by combining it with another powerful spectroscopic technique: $\mathrm{x}$-ray magnetic circular dichroism (XMCD). With XMCD, contributions from individual elements can be isolated and examined, ${ }^{5}$ and the spin and orbital moment can be extracted via use of sum-rule analyses. ${ }^{6}$ These attributes make XMCD a particularly useful technique for examining alloys and other complex magnetic materials such as oxides, ${ }^{7}$ multilayers, ${ }^{8}$ diluted magnetic semiconductors, ${ }^{9}$ and molecular compounds. ${ }^{10}$

The combination of XMCD and FMR, often referred to as x-ray detected ferromagnetic resonance (XFMR), has gained increasing popularity. ${ }^{11,12,14-16}$ Several different modes have been employed to record XFMR spectra. In one approach, time averaged and element-specific FMR spectra have been recorded using microwave excitations that are asynchronous with the photon bunches. ${ }^{11-13}$ A main advantage of this approach is that the full frequency space is accessible as no phase relationship needs to be maintained between the microwave source and the x-ray bunches. An alternative approach takes advantage of the sub-ns X-ray pulses available at most modern $\mathrm{x}$-ray facilities to probe the orbit of precessing magnetic moments stroboscopically as a function of the phase

\footnotetext{
${ }^{\text {a)} P r e s e n t ~ a d d r e s s: ~ S w i s s ~ L i g h t ~ S o u r c e, ~ P a u l ~ S c h e r r e r ~ I n s t i t u t, ~} 5232$ Villigen PSI, Switzerland. Electronic mail: peter.warnicke@psi.ch.
}

between the excitation of the magnetization dynamics and the $\mathrm{x}$-ray bunches. ${ }^{15,16}$ In this approach, a main distinction is the type of source used to excite magnetization dynamics. Pulsed current sources with fast ( $\sim 100 \mathrm{ps})$ rise times can be used to provide a short Oersted field, which couples to the magnetization in a thin film sample. ${ }^{14,17}$ The resulting motion can be characterized by a relatively large angular deviation of the magnetization $\left(\sim 20^{\circ}\right.$ or more $)$ before the magnetization decays in an oscillatory fashion back to the equilibrium position. In principle, any resonance frequency of the system is accessible by tuning an external magnetic bias field.

With the pulsed excitation approach, the timing resolution, which ultimately determines the ability to distinguish phase differences between dissimilar magnetic moments, depends primarily on factors such as the timing jitter $\left(t_{j}\right)$ between the pulser and the bunch clock and the width of the $\mathrm{x}$-ray bunches $\left(\tau_{\gamma}\right)$. To date, timing resolution in the order of $\pm 45 \mathrm{ps}$ has been achieved, which is comparable to the bunch width used in the measurements. ${ }^{17,18}$

As mentioned, another method to implement XFMR is to use continuous microwave excitations that are synchronized (phase locked) with the photon bunches. The technique offers a number of advantages, including low jitter and wide range of excitation angles, enabling studies of the magnetic system in the low angle $\left(<1^{\circ}\right)$ continuous precession mode up to the larger angle, non-linear regime. Moreover, XMFR with synchronized microwave excitations adds the ability to detect the phase of precessing magnetic moments relative to the driving field. ${ }^{19}$ The latter characteristic is of particular importance as it can be used to examine small differences in the phase of precessing elemental moments, which in turn can reveal weak coupling between dissimilar moments. ${ }^{20}$ Another advantage is the ability to measure the element-specific complex magnetic susceptibility $\left(\chi=\chi^{\prime}+i \chi^{\prime \prime}\right)$ at arbitrary admixture between the in-phase $\left(\chi^{\prime}\right)$ and out-of-phase $\left(\chi^{\prime \prime}\right)$ response. ${ }^{15,16,21,22}$ 
In conventional FMR, a wide frequency range for measurement of the resonant response is useful as it enables assessment of the frequency response at microwave bands that are of technological relevance (X-band and beyond). Also, conventional FMR at high frequencies can reveal new physics, such as the contribution of non-Gilbert damping in thin ferromagnetic films. ${ }^{23}$ It is, therefore, useful to examine the potential limiting factors of XFMR such as the time structure of the probe. Of course, additional factors can limit the practical frequency limit of XFMR, such as noise in the detection circuitry (e.g., voltage noise). However, this quantity is specific to the experimental apparatus used to measure the XFMR signal and hence in this paper we focus our investigation on the more general consideration of the timing system, parameterized by $t_{j}$ and $\tau_{\gamma}$, and analyze how these parameters influence the upper accessible frequency of XFMR. We begin by reviewing the experimental methods used in the measurements and then develop a numerical model to explore the effects of $t_{j}$ and $\tau_{\gamma}$.

\section{METHODS}

The measurements were carried out at the I1011 soft $\mathrm{x}$-ray beam line, located on the MAX-II storage ring at MAX-lab, Lund University, Sweden. MAX-II operates with a fundamental bunch clock of $100 \mathrm{MHz}$. The X-ray source for I1011 beam line is an elliptically polarizing undulator, which permits selection of circularly or linearly polarized soft $\mathrm{x}$-rays for experiments. The beam line is also equipped with an eight-pole vector magnet (octupole magnet). The poles of the magnet are oriented along the body centered directions of a conventional cubic geometry, and the maximum gap between the poles is small $(22 \mathrm{~mm})$. The magnetic arrangement provides on-sample magnetic fields $(H)$ up to $\sim 0.5 \mathrm{~T}$ in an arbitrary direction $(\hat{H})$. The vector magnet is particularly useful for XFMR as it permits a simple change of $\hat{H}$ from (i) partially parallel to the photon direction, which is necessary for conventional XMCD scans, to (ii) orthogonal to both the photon direction and the microwave excitation, suitable for phase-resolved XMFR measurements. A photodiode mounted in the octupole chamber is used to detect the photon transmitted through the thin-film samples.

Our microwave circuitry is similar to the one presented by Arena et al. in $2009 .{ }^{16}$ Here, we briefly review the main components of the measurement system, which affect the signal levels and timing measurements. The harmonic spectrum of the bunch clock from the synchrotron is generated by a low phase-noise comb generator and the desired frequency for the XFMR measurements is selected by a narrow band pass filter (BPF). This method restricts the available frequencies to the harmonics of the fundamental x-ray bunch clock, but results in a phase resolution which is considerably smaller than the bunch length of the $\mathrm{x}$-rays. The relatively low frequency of the MAX-II storage ring $(100 \mathrm{MHz})$ correspondingly results in a fine spacing in the harmonic spectrum. Under normal operation, the full width at half maximum (FWHM) of the longitudinal photon bunch length amounts to $\tau_{\gamma}=650$ ps. Timing control is obtained by a digital delay generator located on the high-frequency side of the circuit, which permits variation of the delay time between the microwave pump and the $\mathrm{x}$-ray probe in steps of $5 \mathrm{ps}$. A final amplification stage in the microwave circuit increases the power of the singlefrequency signal to about $+30 \mathrm{dBm}$ before it is directed on to a co-planar waveguide (CPW) where the sample is mounted.

Alternating magnetic fields inductively generated from the microwaves pass through the CPW and excite the magnetization precession in the sample. Under forced precession, the magnetization follows a highly elliptical orbit defined by the effective field, which consists of contributions from the applied bias, anisotropy, and dipolar fields. Our samples have negligible anisotropy and the magnetic easy axis lies in-plane due to the dipolar fields; hence, the in-plane to out-of-plane axis ratio of the precessional orbit is $\gg 1$. As the projection of the XMCD is measured along the propagation direction of the $\mathrm{x}$-ray photons, the alignment of the sample surface with respect to the incoming photons, therefore, determines the XMCD intensity. To increase the component along the photon propagation direction from the magnetization precession, the sample was rotated $20^{\circ}$ away from normal incidence. As the photons arrive at the detector at a rate of $100 \mathrm{MHz}$, we stroboscopically sample the instantaneous projection of the magnetization along the photon beam direction.

XFMR can be used to examine complex structures comprising one or more magnetic elements in several layers. In this study, we examine a trilayer sample consisting of two magnetic layers with in-plane easy axes separated by a nonmagnetic layer. The sample with a layer structure of $\mathrm{Ni}_{81} \mathrm{Fe}_{19}(15) / \mathrm{Cu}(10) / \mathrm{Co}_{93} \mathrm{Zr}_{7}(4) / \mathrm{Cu}(2)$ (thickness in $\mathrm{nm}$ and $\mathrm{Ni}_{81} \mathrm{Fe}_{19}$ as the bottom layer) was deposited onto a $1 \mathrm{~mm} \times 1 \mathrm{~mm}, 100 \mathrm{~nm}$ thick, silicon nitride membrane under UHV base pressure using magnetron sputtering. The sample was mounted with the film side facing a shorted CPW. A perforated hole in the current-conducting central strip-line of the CPW enables transmission of $\mathrm{x}$-rays.

Two measurement protocols were used in the experiments. Field scans are performed by sweeping the magnetic bias field at a constant delay time while recording the XMCD intensity. By selecting an energy corresponding to an elemental core-level, this approach measures the element-specific complex susceptibility, containing linear combinations of $\chi^{\prime}$ and $\chi^{\prime \prime}$, with relative amplitudes chosen through the delay. ${ }^{16}$ Timing delay scans, on the other hand, are performed by scanning the delay time at a fixed bias field and photon energy and such scans can provide a direct mapping of the magnetization precession orbit in the sample.

\section{RESULTS}

Conventional XMCD spectra were measured in transmission mode using magnetic field switching. Fig. 1 shows x-ray absorption spectra (XAS) over the $L_{3}$ and $L_{2}$ edges of $\mathrm{Fe}$ recorded using a fixed helicity of the x-rays. Below the $\mathrm{XAS}$, the XMCD difference spectrum is presented. Under static conditions, the magnetization makes a complete reversal in a single XMCD measurement. A different situation applies under continuous excitation where the precession angle is small: typically, the magnetization cone angle is in the order of $1^{\circ} .{ }^{20}$ Consequently, the XMCD signal intensity 


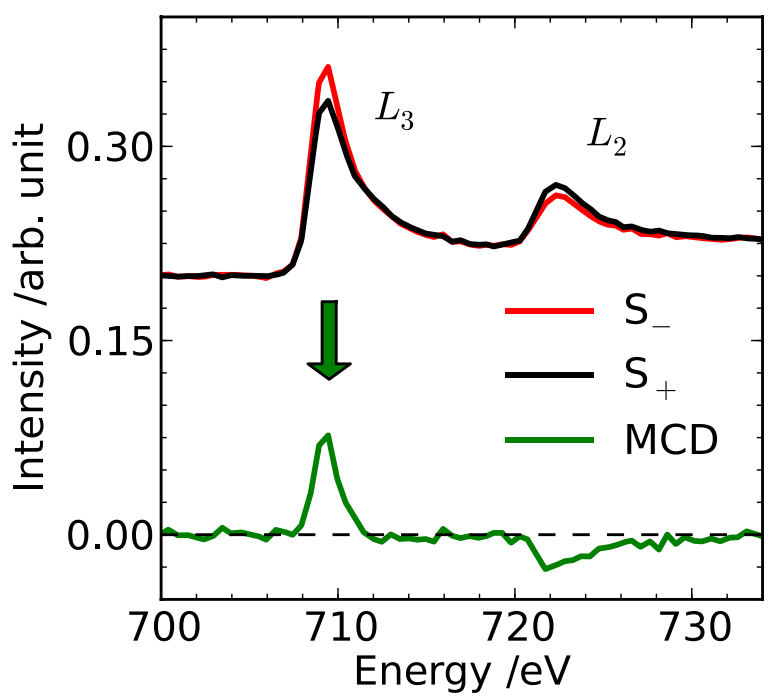

FIG. 1. X-ray spectroscopy for Fe recorded at reversed magnetic polarities $S_{-}$and $S_{+}$. The XMCD is obtained from the difference of these spectra. Time-resolved XMCD scans were taken at the energy marked by the arrow.

is about two orders of magnitude smaller in precessional mode compared to static full-switching mode.

For the forced precession measurements acquired under microwave excitation, we tune the energy to the point of maximum spin asymmetry which maximizes the XMCD signal (see arrow in Fig. 1). Field scans, obtained with a fixed helicity, are presented in Fig. 2(a). In these scans, we measure the amplitude of the magnetization projected along the beam direction as the external field is swept through resonance. The line shape of the field scan is determined by the mixture between $\chi^{\prime}$ and $\chi^{\prime \prime}$, and the relative contribution can be selected by tuning the phase delay between the microwave driving field and the photon bunch clock. As we change the frequency, and thereby the wavelength of the microwaves, we expect a shift in the relative phase. The amplitude as well as the relative phase can be recovered from a fit of the field scan with a complex Lorentzian. In Fig. 2(a), as the frequency is increased from 1.0 to $2.3 \mathrm{GHz}$, we observe a positive shift in the resonance field in accordance with conventional FMR. ${ }^{2}$ The recovered phases of the displayed 1.0, 1.2, 1.3, 1.7, 2.0, and $2.3 \mathrm{GHz}$ field scans are $84^{\circ}$, $68^{\circ}, 67^{\circ}, 98^{\circ}, 102^{\circ}$, and $111^{\circ}$, respectively, indicating that the scans are not purely imaginary but contain also some real part. The square of the resonance frequency is plotted against applied field in Fig. 2(b). Assuming the only significant contribution to the effective field is the applied bias field $H$ (i.e., neglecting anisotropy and contributions from exchange coupling between the layers) our data are well described by the Kittel relation ${ }^{2}$

$$
f^{2}=\left(\mu_{0} \gamma /(2 \pi)\right)^{2} H_{\text {res }}\left(H_{\text {res }}+M_{s}\right)
$$

at resonance $H=H_{\text {res }}$, where $\mu_{0}$ is the vacuum permeability and $\gamma$ the gyromagnetic ratio. From the fit, we extract a saturation magnetization $\mu_{0} M_{s}=1.0 \mathrm{~T}$, which is in line with expected values for $\mathrm{Ni}_{81} \mathrm{Fe}_{19} \cdot{ }^{24}$

As mentioned, an alternative way to probe the precessional motion is to stroboscopically sample the direction of the
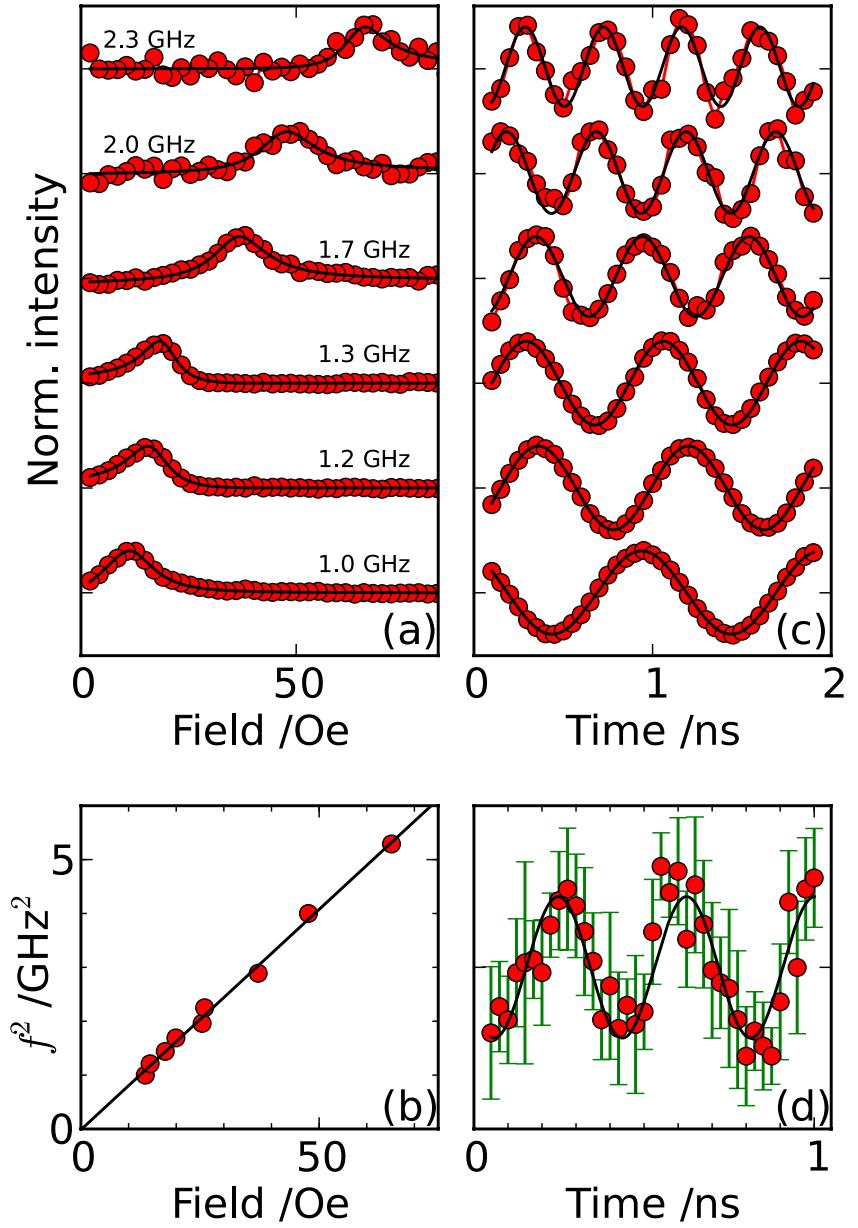

FIG. 2. XFMR measurements. (a) Field scans of the normalized XMCD intensity showing data as solid circles and complex Lorentzian fits as solid lines. (b) Resonance frequency squared plotted against field. (c) Time delay scans of the normalized XMCD intensity (solid circles) obtained at the resonance field with sinusoidal fits (solid line). (d) Time delay scan at $2.5 \mathrm{GHz}$ averaged over 5 scans at a bias field of 78 Oe. The vertical bars are standard deviations to the data.

magnetization as a function of the delay time between the x-ray pulses and the microwave excitation, and such timing delay scans are presented in Fig. 2(c). At a fixed frequency and near the resonant field, the timing scans have a maximum amplitude, which decays away as the field deviates from the resonant value. As expected, the resonant amplitude is reduced as the frequency is increased, indicating that the opening angle of the precession cone decreases with increasing frequency at a constant power. At frequencies above $2.3 \mathrm{GHz}$, the noise level of the field scans becomes comparable with the signal level and field scans similar to the data presented in Fig. 2(a) reveal only noise. In contrast, timing scans performed at an extrapolated resonance field value reveal discernible oscillations at a driving frequency of up to $2.5 \mathrm{GHz}$, as seen in Fig. 2(d). This is the central result of our paper. It is clear that the observed oscillation period (400 ps) is considerably shorter than the x-ray bunch length from the synchrotron ( $650 \mathrm{ps})$.

To address the experimental factors on the timing side of the detection circuit, which limit the accessible frequency range, we construct a numerical model for the sampling process. We will assume that, in analogy with a forced harmonic oscillator, the magnetization is driven to forced precession 
and attains the same frequency $f$ as the magnetic driving field. The projection of the precessing magnetization onto the vector of the incoming $\mathrm{x}$-rays for a given driving frequency $f$ can be represented by $s(t)=A_{0} \sin (2 \pi f t+\delta)$ with a phase $\delta$. The coefficient $A_{0}$ for a forced harmonic oscillator can be described by

$$
A_{0} \propto \frac{1}{\sqrt{4 \pi^{2}\left(f_{0}^{2}-f^{2}\right)^{2}+\lambda^{2} f^{2}}},
$$

where $f_{0}$ is the resonance frequency and $\lambda$ a damping parameter. ${ }^{19}$ At resonance, this expression reduces to $A_{0} \propto 1 / f$ assuming the damping is constant. The model further assumes that the $\mathrm{X}$-ray probes, $p(t)$, are uniform, Gaussian pulses

$$
p(t)=\frac{1}{\sigma_{\gamma} \sqrt{2 \pi}} e^{-t^{2} /\left(2 \sigma_{\gamma}^{2}\right)}
$$

with a FWHM of $\tau_{\gamma}=2 \sqrt{2 \ln 2} \sigma_{\gamma}$. We simulate a delay scan at a fixed frequency $f$ numerically by evaluating the integrated product of the precession orbit with a sampling pulse, accumulated over $N$ photon bunches
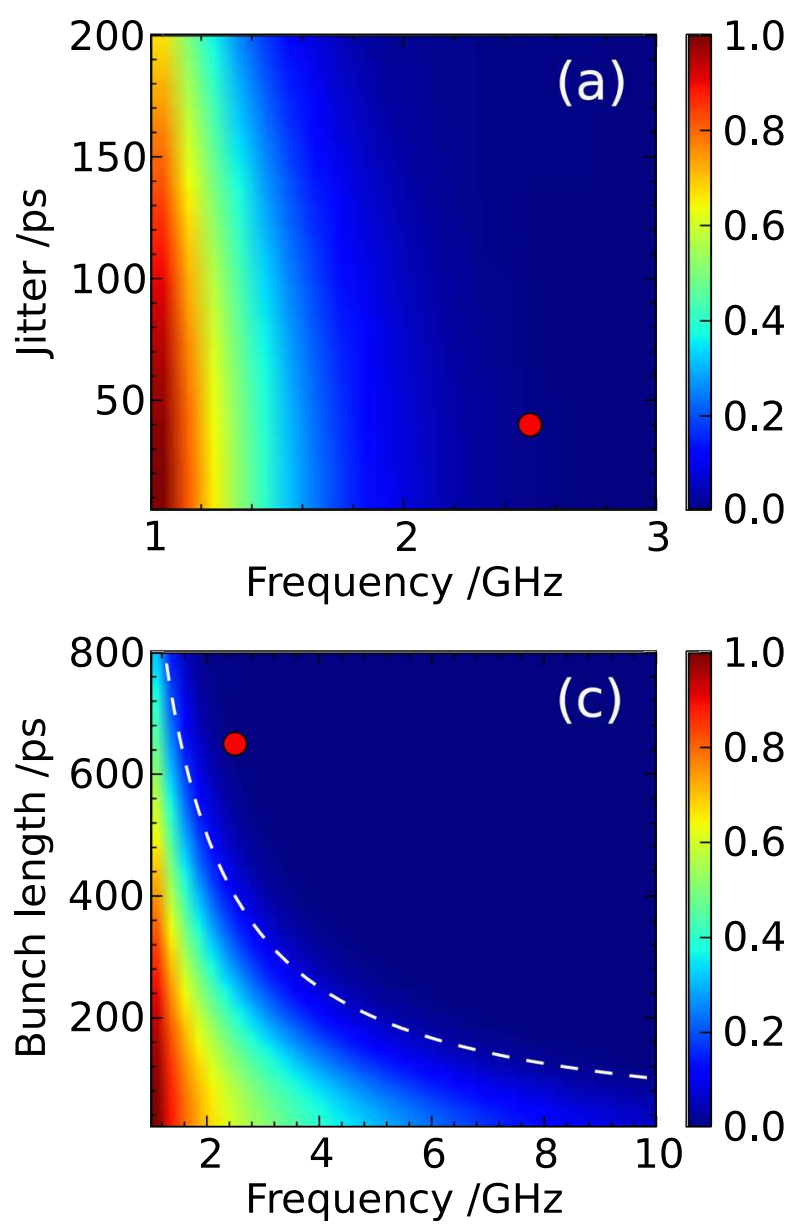

$$
S\left(\tau_{\gamma}, \sigma_{j}, t_{d}, f\right)=\frac{1}{N} \sum_{n=1}^{N} \int_{-\infty}^{\infty} s(t) p\left(t-t_{d}-t_{j}(n)\right) d t
$$

for each delay time $t_{d}$ in the time range. ${ }^{25}$ To take into account the jitter of the bunch clock, the timing of the probe for each sampling event $n$ is shifted in time by a Gaussian distributed random number $t_{j}$ with a standard deviation $\sigma_{j} .{ }^{28}$ In accordance with the experimentally measured amplitude, the numerically sampled amplitude $A$ is extracted from a fit to the simulated delay scan. In Fig. 3, we compare the numerical calculations of the amplitude with experimental data.

The influence of timing jitter and frequency on amplitude at a fixed bunch length is investigated in Fig. 3(a). At any given point in the parameter space, the amplitude drops monotonically with increasing $f$ or $\sigma_{j}$. In relative terms, the amplitude is more sensitive to a change of $f$ characterized by a slope more than two orders of magnitude larger than for $\sigma_{j}$ at the position of the solid circle. The shape of the amplitude landscape can be understood by noting that the jitter produces a shift of each probe, which results in a net broadening and reduction of the sampled intensity. As seen in Eq. (2), there is an increase in the precessional orbit amplitude for lower frequencies due to the $1 / f$ dependence.
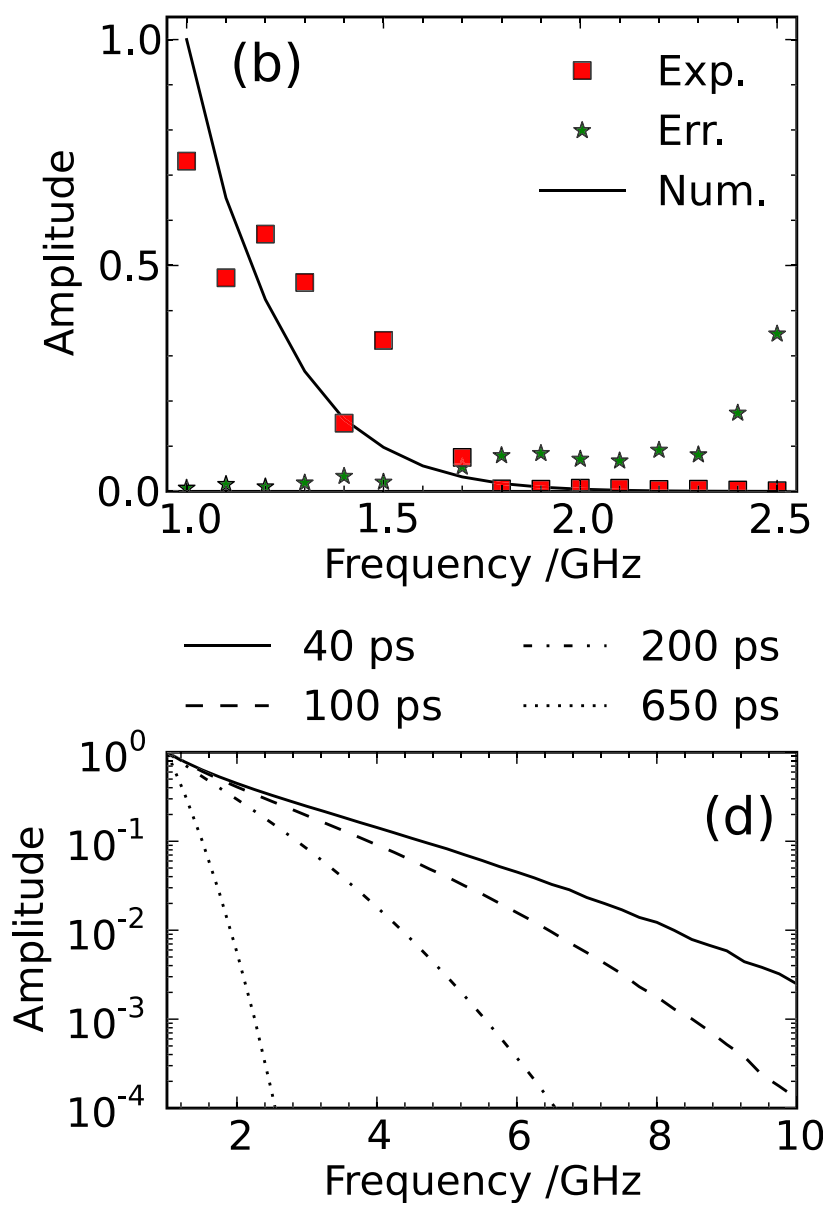

FIG. 3. Numerical amplitude calculations compared with experiment. (a) For visual clarity, the square-root of the normalized amplitude $A$ is plotted versus $\sigma_{j}$ and $f$ for a fixed bunch length of $\tau_{\gamma}=650 \mathrm{ps}$. The solid circle marks the experimental conditions of the highest attainable frequency at MAX-lab. (b) Experimental and numerical amplitudes of the timing scans, both following a decaying trend with increasing frequency. The standard deviation of the fits to the experimental data is normalized with the amplitude (star symbols). (c) Square-root of the normalized amplitude plotted versus $\tau_{\gamma}$ and $f$ for a fixed jitter of $\sigma_{j}=40 \mathrm{ps}$. A dashed line marks the $1 / f$ bunch length for comparison. (d) Amplitude profiles for 40 to $650 \mathrm{ps}$ bunch length at $\sigma_{j}=40 \mathrm{ps}$. 
The experimental amplitude decays to a frequency region where the relative noise is about one order of magnitude larger. This is illustrated in Fig. 3(b) together with numerical data using parameters corresponding to the experimental conditions: $\tau_{\gamma}=650 \mathrm{ps}$ and $\sigma_{j}=40 \mathrm{ps}^{26}$ The numerical amplitude follows a non-linear decaying trend which is also observed in the experimental data. Comparing the fitting error of the measured timing scans with the numerical timing scans two regimes are present in the frequency spectrum. For $f \leq 2.3 \mathrm{GHz}$, the fitting error of the experimental data (star symbols) increases linearly with a slope of $\sim 0.14 \mathrm{GHz}^{-1}$ followed by a sharp increase in slope to $1.8 \mathrm{GHz}^{-1}$ for $f>2.3 \mathrm{GHz}$. The numerical fits follow a similar trend with a cut-off frequency at about $2.6 \mathrm{GHz}$ (not shown). The relatively small discrepancy in the fitting error for the amplitude between the experimental data and the simulated delay scans may be due to the voltage noise in the measurement, which is not included in our modeling.

Keeping the jitter parameter constant at $40 \mathrm{ps}$, the influence of the photon bunch length $\tau_{\gamma}$ is examined in Fig. 3(c). Again, a monotonic decay in amplitude is observed for increasing $f$ or $\tau_{\gamma}$. At a fixed frequency, the amplitude is sensitive to a relative change in $\tau_{\gamma}$. For example, a reduction of the bunch length from $650 \mathrm{ps}$ to $550 \mathrm{ps}$ leads to an increase in the sampled amplitude from $3.3 \times 10^{-5}$ to $4.9 \times 10^{-4}$. At the same position, the gradients have the same order of magnitude along $f$ and $\tau_{\gamma}$ implying that the amplitude is more sensitive to a change in $\tau_{\gamma}$ as compared to a change in $\sigma_{j}$.

In Fig. 3(d), the amplitude decay is shown as a function of frequency for different choices of the bunch length and at a constant jitter of $40 \mathrm{ps}$. At a bunch length of $650 \mathrm{ps}$, the relative amplitude crosses the $10^{-4}$ limit at about $2.5 \mathrm{GHz}$, which coincides with the frequency where signals are still discernible in the experiments (see Fig. 2). Using this value as an estimate for the detection limit, the upper detectable frequency increases at shorter bunch lengths. For 100 ps photon bunches (available at several synchrotrons), the upper detectable frequency exceeds $10 \mathrm{GHz}$. Detection at higher frequencies can be a practical challenge due to prominent noise. The signal-to-noise ratio scales with the square root of the number of probing events so to maintain the signal level at $10 \%$ of the amplitude one needs to increase the measurement time by a factor 100 . The choice of a constant jitter in Fig. 3(d) focuses the analysis of the XFMR frequency limit on the bunchlength. It should be noted that the jitter is also expected to influence the upper detectable frequency.

\section{DISCUSSION}

The experimental data and the numerical analysis imply that the accessible frequency range in phase-resolved XFMR extends well beyond $1 / \tau_{\gamma}$ (this ratio amounts to $1.54 \mathrm{GHz}$ at the MAX-II storage ring). While the amplitude of the signal decays rapidly for increasing frequencies above this point, the field scans can be measured at considerably higher frequencies and the timing scans have a detectable signal at even higher frequencies. These results suggest that it is feasible to extend the frequency range of phase-sensitive and $\mathrm{x}$-ray detected FMR beyond the $\mathrm{X}$-band, especially in consideration to the $<80 \mathrm{ps}$ bunch lengths available at several of today's third generation synchrotrons. Furthermore, experimental schemes to transferring the XFMR technique to fourth generation synchrotrons, where photon bunch lengths in the sub-ps regime can be achieved, have recently been proposed. ${ }^{27}$

In conclusion, experimental measurements of the upper accessible frequency for transmission mode XFMR exceed the inverse bunch length. The observed upper accessible frequency is supported by a numerical model, which accounts for the sampled amplitude dependence on the pump frequency as well as the time structure of the probe, i.e., the bunch length and timing jitter. The model could be extended further by including additional sources of jitter, for example, sampling noise ${ }^{28}$ originating from the detector side of the circuit.

\section{ACKNOWLEDGMENTS}

The authors thank Dr. Gunnar Öhrwall at MAX-lab for beamline support. The support of the Swedish Foundation for International Cooperation in Research and Higher Education (STINT) is gratefully acknowledged. The support of the NSLS under DOE Contract No. DE-AC02-98CH10886 is also gratefully acknowledged. W.E.B. acknowledges support from the U.S. NSF-ECCS-0925829.

${ }^{1}$ J. H. E. Griffiths, Nature 158, 670 (1946).

${ }^{2}$ C. Kittel, Phys. Rev. 73, 155 (1948).

${ }^{3}$ M. Sparks, Ferromagnetic Relaxation Theory (McGraw-Hill, New York, 1964).

${ }^{4}$ M. Farle, Rep. Prog. Phys. 61, 755 (1998).

${ }^{5}$ G. Schütz, W. Wagner, W. Wilhelm, P. Kienle, R. Zeller, R. Frahm, and G. Materlik, Phys. Rev. Lett. 58, 737 (1987).

${ }^{6}$ C. T. Chen, Y. U. Idzerda, H.-J. Lin, N. V. Smith, G. Meigs, E. Chaban, G. H. Ho, E. Pellegrin, and F. Sette, Phys. Rev. Lett. 75, 152 (1995).

${ }^{7}$ M. Besse, V. Cros, A. Barthélémy, H. Jaffrès, J. Vogel, F. Petroff, A. Mirone, A. Tagliaferri, P. Bencok, P. Decorse, P. Berthet, Z. Szotek, W. M. Temmerman, S. S. Dhesi, N. B. Brookes, A. Rogalev, and A. Fert, Europhys. Lett. 60, 608 (2002).

${ }^{8}$ D. Weller, Y. Wu, J. Stöhr, M. G. Samant, B. D. Hermsmeier, and C. Chappert, Phys. Rev. B 49, 12888 (1994).

${ }^{9}$ M. Kobayashi, Y. Ishida, J. 1. Hwang, T. Mizokawa, A. Fujimori, K. Mamiya, J. Okamoto, Y. Takeda, T. Okane, Y. Saitoh, Y. Muramatsu, A. Tanaka, H. Saeki, H. Tabata, and T. Kawai, Phys. Rev. B 72, 201201 (2005).

${ }^{10}$ A. Scheybal, T. Ramsvik, R. Bertschinger, M. Putero, F. Nolting, and T. A. Jung, Chem. Phys. Lett. 411, 214 (2005).

${ }^{11}$ G. Boero, S. Rusponi, P. Bencok, R. S. Popovic, H. Brune, and P. Gambardella, Appl. Phys. Lett. 87, 152503 (2005).

${ }^{12}$ J. Goulon, A. Rogalev, F. Wilhelm, N. Jaouen, C. Goulon-Ginet, G. Goujon, J. Ben Youssef, and M. V. Indenbom, JETP Lett. 82, 696 (2005).

${ }^{13}$ G. Boero, S. Rusponi, J. Kavich, A. Lodi Rizzini, C. Piamonteze, F. Nolting, C. Tieg, J.-U. Thiele, and P. Gambardella, Rev. Sci. Instrum. 80, 123902 (2009).

${ }^{14}$ S. Buschhorn, F. Brüssing, R. Abrudan, and H. Zabel, J. Phys. D 44, 165001 (2011).

${ }^{15}$ M. K. Marcham, P. S. Keatley, A. Neuder, R. J. Hicken, S. A. Cavill, L. R. Shelford, G. van der Laan, N. D. Telling, J. R. Childress, J. A. Katine, P. Shafer, and E. Arenholz, J. Appl. Phys. 109, 07D353 (2011).

${ }^{16}$ D. A. Arena, Y. Ding, E. Vescovo, S. Zohar, Y. Guan, and W. E. Bailey, Rev. Sci. Instrum. 80, 083903 (2009).

${ }^{17}$ W. E. Bailey, L. Cheng, D. J. Keavney, C.-C. Kao, E. Vescovo, and D. Arena, Phys. Rev. B 70, 172403 (2004).

${ }^{18}$ S. Buschhorn, F. Brüssing, R. Abrudan, and Hartmut Zabel, J. Synchrotron Radiat. 18, 212 (2011).

${ }^{19}$ Y. Guan, W. E. Bailey, E. Vescovo, C. C. Kao, and D. A. Arena, J. Magn. Magn. Mater. 312, 374 (2007). 
${ }^{20}$ D. A. Arena, E. Vescovo, C.-C. Kao, Y. Guan, and W. E. Bailey, Phys. Rev. B 74, 064409 (2006).

${ }^{21}$ T. Martin, G. Woltersdorf, C. Stamm, H. A. Dürr, R. Mattheis, C. H. Back, and G. Bayreuther, J. Appl. Phys. 105, 07D310 (2009).

${ }^{22}$ P. Klaer, F. Hoffmann, G. Woltersdorf, E. Arbelo Jorge, M. Jourdan, C. H. Back, and H. J. Elmers, J. Appl. Phys. 44, 425004 (2011).

${ }^{23}$ J. Lindner, K. Lenz, E. Kosubek, K. Baberschke, D. Spoddig, R. Meckenstock, J. Pelzl, Z. Frait, and D. L. Mills, Phys. Rev. B 68, 060102 (2003).

${ }^{24}$ G. Counil, J.-V. Kim, T. Devolder, C. Chappert, K. Shigeto, and Y. Otani, J. Appl. Phys. 95, 5646 (2004).

${ }^{25}$ For the numerically calculated timing scans, a total range of $2 \mathrm{~ns}$ is used with a time delay interspacing of $10 \mathrm{ps}$. The number of probing events per data point was kept at $N=1000$, where the data showed good convergence. In the experimental delay scans, the number of photon bunches per data point was typically $10^{8}$

${ }^{26}$ The experimental jitter was measured using a digital sampling oscilloscope and defined as the $\sigma$ of approximately 10000 excitation events triggered with respect to the bunch clock. It should be noted that the timing jitter depends on the components in the microwave circuit and may therefore vary between similar setups at the same experimental station.

${ }^{27}$ A. Rogalev, J. Goulon, G. Goujon, F. Wilhelm, I. Ogawa, and T. Idehara, J. Infrared Millim. Terahertz Waves 33(7), 777-793 (2012).

${ }^{28}$ M. Shinagawa, Y. Akazawa, and T. Wakimoto, J. Solid-State Circuits 25, 220 (1990). 